\title{
Neuralgische Schulteramyotrophie: Eine extrahepatische Manifestation der Hepatitis E
}

\author{
Sven Pischke, Ulrike Ryll, Andres De Weerth, Friederike Ufer, Mathias Gelderblom
}

Die neuralgische Schulteramyotrophie ist durch akute starke neuropathische Schmerzen im Schulter-Arm-Bereich gekennzeichnet. Nachfolgend entwickeln sich multifokale Paresen und Atrophien im Versorgungsgebiet einzelner oder mehrerer Arm-Nerven. In seltenen Fällen kann diesem Krankheitsbild eine Hepatitis E zugrunde liegen - wie im diesem Fall eines 46-jährigen Patienten.

Schmerzen in den Schultern I Ein 46 Jahre alter Mann (keine Vorerkrankungen bis auf arterielle Hypertonie, Nichtraucher, gelegentlicher Alkoholkonsum) wird in einer Notaufnahme vorstellig, weil er unter stärksten Schmerzen beidseits in den Schultern leidet. Die Schmerzen sind nachts aufgetreten und haben einen brennend stechenden Charakter. Nach 3-4 Tagen habe die Intensität nachgelassen. In der Folge habe er eine unscharf begrenzte Hypalgesie und Hypästhesie im Bereich des rechten Unteram entwickelt und Myalgien im Bereich des M. biceps brachii, M. brachioradialis rechts und M. biceps brachii links. Höhergradige Paresen seien subjektiv nicht aufgefallen, allerdings habe er den Arm schmerzbedingt geschont.

Blutwerte I Im Rahmen der Blutentnahme in der Notaufnahme zeigen sich deutlich erhöhte Werte für AST (max. 495U/1) und ALT (max. 1152U/1) ( $\triangleright$ Abb. 1). Die weitere Diagnostik schließt eine Virushepatitis $\mathrm{A}-\mathrm{C}$ aus, doch serologisch kann eine Hepatitis E diagnostiziert werden (Mikrogen-Assays, Mikrogen, Neuried Deutschland). Die Viruslast ist zu diesem Zeitpunkt jedoch ungewiss, da nicht per PCR auf HEV getestet wird. Es erfolgt eine analgetische Behandlung mit Ibuprofen.

Persistierende Schmerzen I Der Hausarzt vermutet einen Zusammenhang zwischen Hepatitis E und den starken Schulterschmerzen und stellt den Patienten daraufhin bei uns vor, da unsere Abteilung aufgrund der Behandlung zahlreicher Hepatitis-E-Fälle über Expertise auf diesem Gebiet verfügt. Auch 16 Tage nach initialer Vorstellung in der Notaufnahme bestehen weiterhin starke Schulterschmerzen.

Neurologische Untersuchung I Im neurologischen Befund stellten sich bei der Vorstellung 4/5 (MRCSkala) schlaffe Paresen im Bereich der rechten Schulter- und proximalen Oberarmmuskulatur dar (Schulter-Abduktion und -Elevation; Ellenbogen-Beuger und -Strecker). Im Bereich der Hand und der Finger fanden sich rechts volle Kraftgrade. Links war isoliert eine schlaffe Parese des M. opponens pollicis feststellbar. Der Tricepssehenreflex war beidseits erloschen, der Bicepssehenreflex war rechts schwach unter Bahnung, links erloschen und der Radiusperiostreflex war beidseits erloschen. Die Muskeleigenreflexe im Bereich der unteren Extremitäten waren symmetrisch lebhaft auslösbar. Muskelatrophien stellten sich nicht dar und die Sensibilität war unauffällig.

Elektrophysiologische Diagnostik | In der elektrophysiologischen Diagnostik fanden sich in der motorischen Neurografie des N. ulnaris normale distal motorische Latenzen, motorische Amplituden (MSAP) und Nervenleitgeschwindigkeiten (NLG). Die F-Wellen des N. ulnaris rechts hatten eine normale Latenz und Persistenz, links war die Latenz normal und die Persistenz mit 10/20 reduziert. Leitungsblöcke stellen sich in den untersuchten Abschnitten nicht dar.

Motorische Teilschädigung | In der sensiblen Neurografie waren die sensiblen Amplituden (SNAP) und die NLG ebenfalls des N. ulnaris bds. unauffällig. In der ergänzenden Elektromyografie stellten sich rechts im M. deltoideus, M. biceps brachii, M. brachioradialis und $M$. interosseus dorsalis manus I die Potenziale muskulärer Einheiten (PME) mit einer erhöhten Polyphasierate

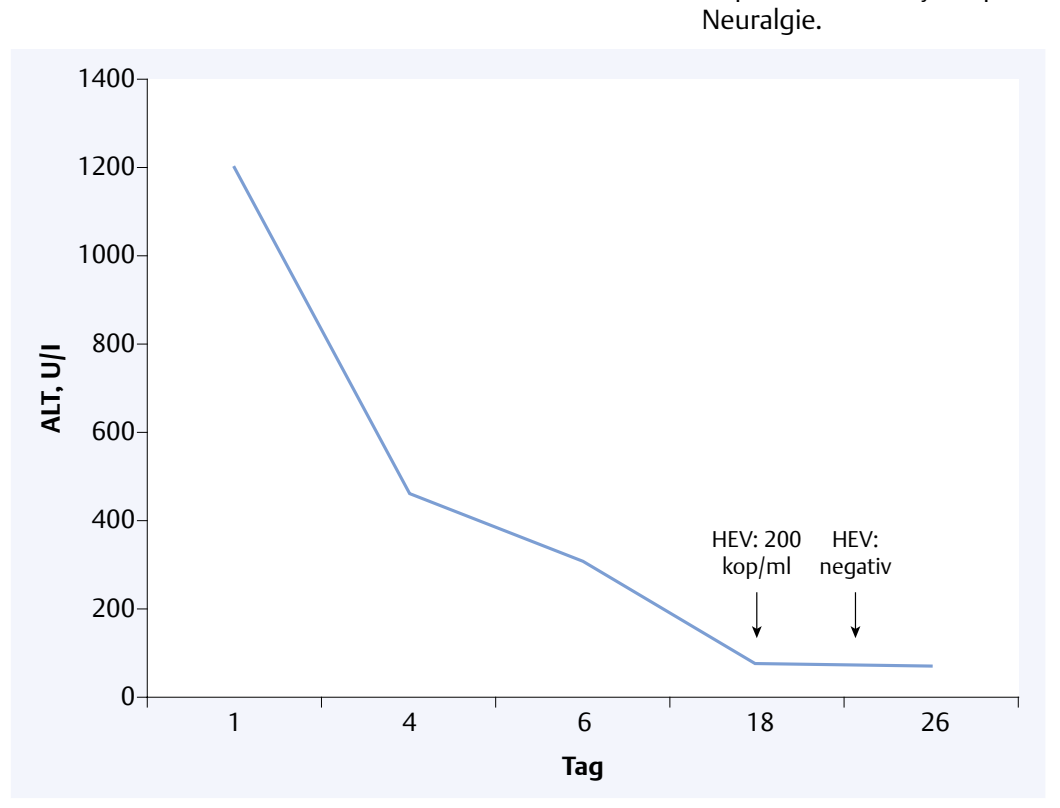

Abb. 1 Verlauf der GPT bei einem Patienten mit Hepatitis E und amyotropher Neuralgie. 
A. Sensible Neurografie des $\mathbf{N}$. ulnaris rechts

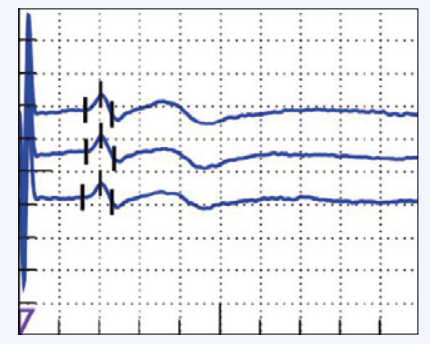

\begin{tabular}{|l|c|c|c|c|c|c|c|c|c|}
\hline Position & Latenz & Amplitude & Dauer & Fläche & Entfg. & Reiz & NLG & Temp. & kNLG \\
\hline Handgelenk & $3,3 \mathrm{~ms}$ & $9,3 \mathrm{uV}$ & $1,3 \mathrm{~ms}$ & $7,3 \mathrm{uVms}$ & $140 \mathrm{~mm}$ & $13 \mathrm{~mA}$ & $42,2 \mathrm{~m} / \mathrm{s}$ & 32,0 & $45,2 \mathrm{~m} / \mathrm{s}$ \\
\hline Handgelenk 1 & $3,4 \mathrm{~ms}$ & $9,5 \mathrm{uV}$ & $1,4 \mathrm{~ms}$ & $8,0 \mathrm{uVms}$ & $140 \mathrm{~mm}$ & $13 \mathrm{~mA}$ & $41,7 \mathrm{~m} / \mathrm{s}$ & 32,0 & $44,7 \mathrm{~m} / \mathrm{s}$ \\
\hline Handgelenk 2 & $3,2 \mathrm{~ms}$ & $8,5 \mathrm{uV}$ & $1,5 \mathrm{~ms}$ & $6,8 \mathrm{uVms}$ & $140 \mathrm{~mm}$ & $13 \mathrm{~mA}$ & $44,0 \mathrm{~m} / \mathrm{s}$ & 32,0 & $47,0 \mathrm{~m} / \mathrm{s}$ \\
\hline
\end{tabular}

B. Motorische Neurografie des $\mathrm{N}$. ulnaris rechts

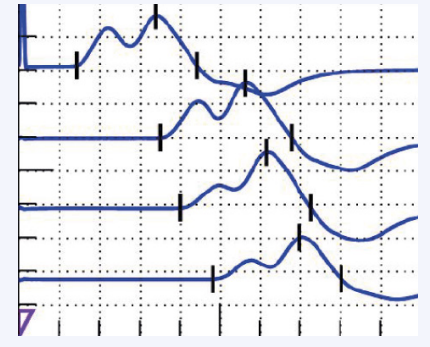

C. F-Wellen des $\mathbf{N}$. ulnaris rechts

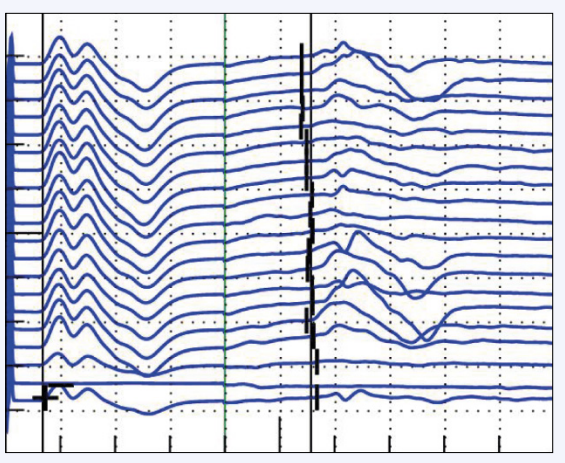

E. EMG des M. brachioradialis rechts

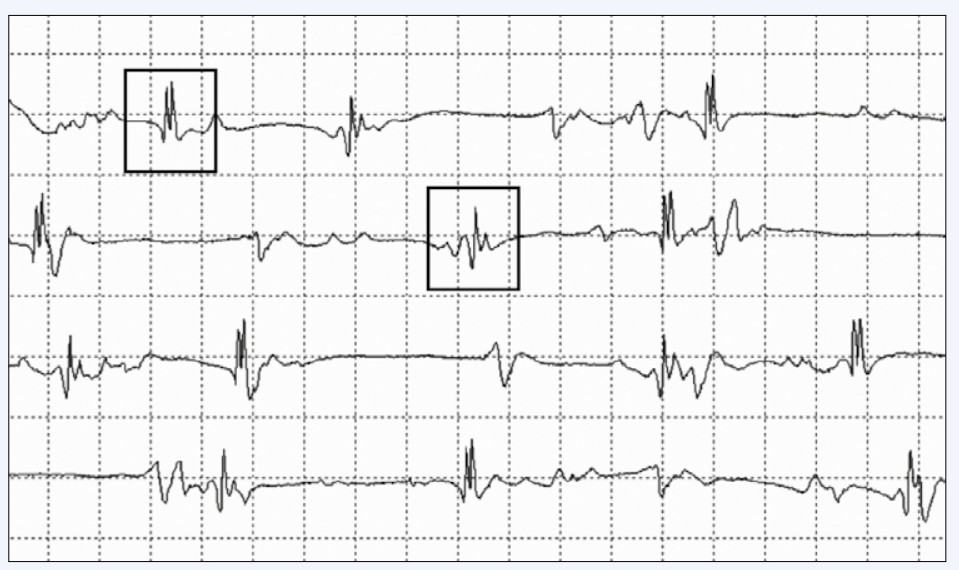

\section{EMG des M. brachioradialis rechts in Ruhe}

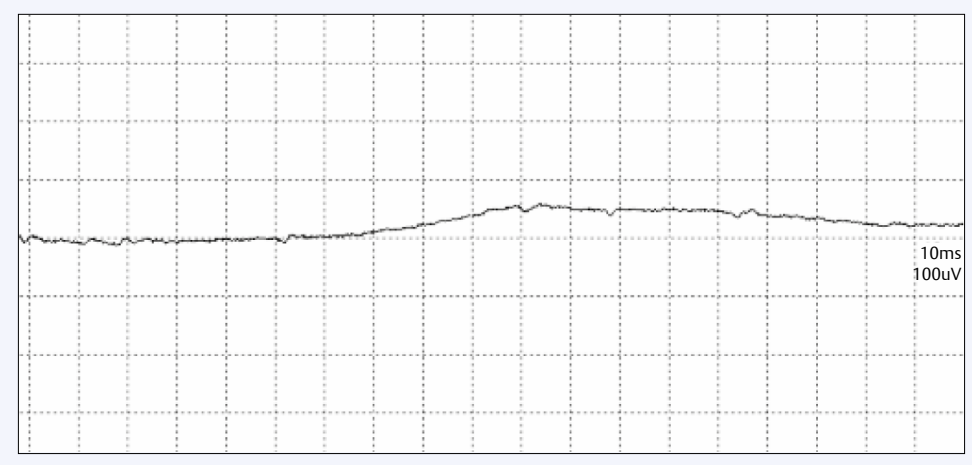

F. EMG des M. brachioradialis rechts

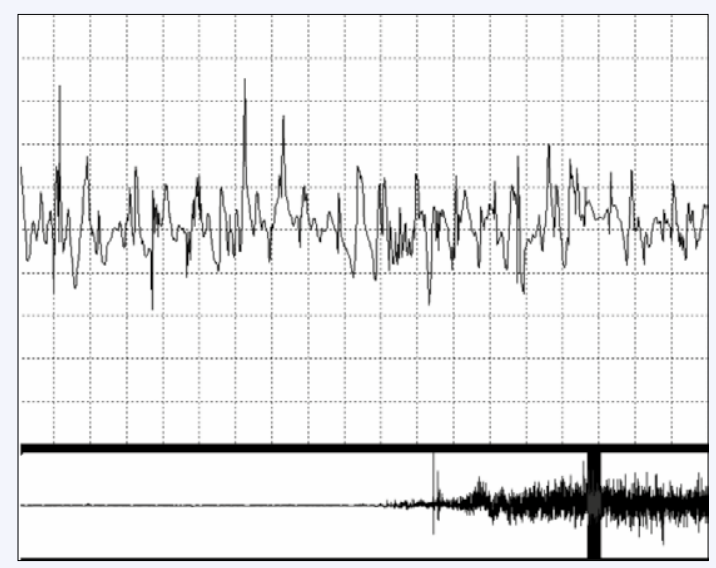

Abb. 2 (A) Sensible Neurografie des N. ulnaris rechts mit normaler distal motorischer Latenz, normalen Amplituden und normaler Nervenleitgeschwindigkeit. (B) Motorische Neurografie des N. ulnaris rechts mit normalen Amplituden und normaler Nervenleitgeschwindigkeit. (C) F-Wellen des N. ulnaris rechts mit unauffälliger Latenz und Persistenz. (D) Elektromyografie (EMG) des rechten M. brachioradialis ohne Hinweise auf pathologische Spontanaktivität, mit (E; markiert mit Kasten) überwiegend polyphasischen Potenzialen motorischer Einheiten und (F) einer dichten Interferenz bei unauffälliger Entladungsfrequenz bei maximaler Willkürinnervation. Entfg: Entfernung; NLG: Nervenleitgeschwindigekeit; Temp: Temperatur; kNLG: temperaturkorrigierte Nervenleitgeschwindigkeit

bei unauffälligen Amplitude und unauffälliger Dauer dar. Es fand sich keine pathologische Spontanaktivität und die Rekrutierung war unauffällig. Zusammenfassend war der elektrophysiologische Befund vereinbar mit Zeichen einer dezenten chronisch neurogenen aktuell rein motorischen Teilschädigung betont im Bereich des Plexus cervicobrachialis rechts unter Einbeziehung aller Faszikel ohne Hinweise auf eine floride Denervierung ( Abb. 2). 
Publikation Inhalt

Fong et al. [22]

Hepatitis E mit NA bei einem Reiserückehrer aus Equador

Rianthavorn et al. [8] Komplette Sequenzierung eines HEV-Genotyp-3-Strangs bei einem Thailänder mit NA

Kamar et al. [14] NA und Hepatitis E, langsame Besserung innerhalb von 18 Monaten

Carli et al. [15] NA und Chorioretinitis bei Patient mit akuter Hepatitis E, behandelt mit Steroidbolus

Inghilleri et al. [16] Hepatitis E mit NA, nach Verzehr einer ungegarten Schweinewurst („Figatellu“)

Moisset et al. [6] Beidseitige NA im Rahmen einer Hepatitis E, zusätzlich Schluckstörungen

Peri et al. [17]

Woolson et al. [18] Spontane Ausheilug einer bilateralen, HEV-assoziierten NA, binnen 3 Monaten

Von 106 Patienten mit Hepatitis E hatten 8 (7,5\%) neurologische Symptome. $3(2,8 \%)$ hatten eine NA.

Deroux et al. [19] Einzelfallbeschreibung: Hepatitis E und NA

Van Eijk et al. [9] $\quad 10,6 \%(n=5)$ von 47 Patienten mit NA hatten serologisch und klinisch eine akute Hepatitis E, bei 4 davon durch PCR bestätigt

Decard et al. [5] MRT- und Sono-morphologischer Nachweis einer Schwellung des Plexus brachialis bei Patientem mit beidseitiger NA und Hepatitis $\mathrm{E}$

Theochari et al. [7] Komplette Remission einer HEV-assoziierten NA binnen 10 Monaten, nach Steroidbehandlung

Dartevel et al. [20] Detaillierte Beschreibung von 5 Fällen von Hepatitis E und NA, sowie detailierte Literatur-Recherche

Rodriguez et al [21] 6 Fälle von NA und Hepatitis E, 2 mit Immunglobulinen und einer mit Steroiden behandelt

Antivirale Therapie I Vor dem Hintergrund der Anamnese, des klinischen Befunds und der elektrophysiologischen Befunde diagnostizieren wir eine neuralgische Plexusamyotrophie, werten diese als assoziiert mit der HEV-Infektion und erwägen, eine antivirale Therapie mit Ribavirin einzuleiten. Da die Transamiansen mittlerweile schon deutlich gefallen sind ( $\triangleright$ Abb. 1 ), gehen wir von einer baldigen Ausheilung der Hepatitis E aus und initialisieren nicht unmittelbar eine Ribavirin-Medikation, sondern warten das PCR-Ergebnis ab. Dieses zeigt eine nur sehr niedrige HEV-Kopienzahl (200 kop/ml, \ Abb. 1). Wir verordneten Pregabalin als zusätzliche Schmerzmedikation.

Weiterer Verlauf | Eine Woche später haben sich in einer Verlaufskontrolle die Beschwerden deutlich gebessert, das Ergebnis der HEV-PCR ist negativ. Die Schmerzmedikation kann beendet werden.

\section{Diskussion}

Charakteristik | Die neuralgische Schulteramyotrophie (NA) wurde erstmals 1943 von Spillane anhand einer Serie von 46 Patienten mit einer „lokalen Neuritis des Schultergürtels“ beschrieben [1]. Gekennzeichnet ist die neuralgische Schulteramyotrophie durch akute starke neuropathische Schmerzen im Schulter-Arm-Bereich mit nachfolgender Entwicklung multifokaler Paresen und Atrophien im Versorgungsgebiet einzelner oder mehrerer Arm-Nerven [2]. Sie betrifft meist die oberen Extremitäten und die Erholung dauert Monate bis Jahre. Der rechte Arm ist häufiger betroffen und eine oft subklinische bilaterale Beteiligung kommt in ca. 33\% der Fälle vor. Die Äste des oberen Armplexus bzw. des posterioren Faszikels (N. thoracicus longus, N. axillaris, N. radialis, N. suprascapularis) werden einzeln oder in Kombination bevorzugt einbezogen. Selten sind isolierte Paresen der distalen Arm-Muskulatur, Affektionen des N. medianus/N. interosseus anterior, N. phrenicus, kaudaler Hirnnerven oder des Plexus lumbosacralis.

Inzidenz | Die Inzidenz der idiopathischen NA beträgt 2-4/100000/Jahr. Männer sind im Verhältnis 3:2 häufiger betroffen und das typische Manifestationsalter liegt im 2. bis 4. Lebensjahrzehnt.

Pathophysiologie I Der exakte pathophysiologische Mechanismus der neuralgischen Schulteramyotrophie ist unklar. Angenommen wird aktuell primär ein immunvermittelter Prozess, der sich gegen periphere Nerven richtet. Dies basiert auf der Beobachtung, dass bei etwa der Hälfte der betroffenen Patienten ein Ereignis, das als Trigger des Immunsystems gelten kann, der Attacke vorausgeht. Hierzu zählen

- Infektionen,

- chirurgische Eingriffe,

- immunmodulatorische Therapien mit Interleukin-2 und Interferon alpha-2.

Als weitere Faktoren, die an der Auslösung von Attacken beteiligt sind, konnten epidemiologische Studien spezifische genetische Faktoren und eine mechanische Vulnerabilität identifizieren.
Tab. 1 Fälle neuralgischer Schulteramyotrophie assoziiert mit Hepatitis E.

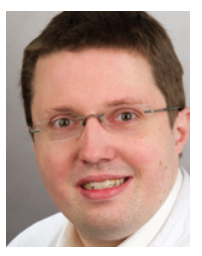

PD Dr. med. Sven Piscke ist Oberarzt am Zentrum für Innere Medizin am UKE Hamburg.

s.pischke@uke.de

Dr. med. Ulrike Ryll ist Ärztin in der Praxis Hoheluftchaussee, Hamburg. klauswinckler@web.de 


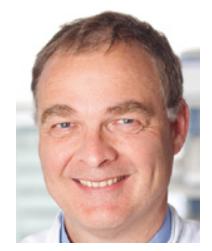

Dr. med. Andres de Weerth ist Chefarzt in der Inneren Medizin am Agaplesion Krankenhaus, Hamburg. deweerth@adew.de

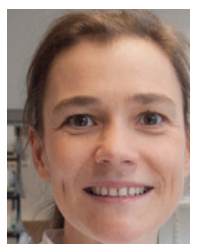

Dr. med. Friederike Ufer ist Assistenzärztin an der Klinik und Poliklinik für Neurologie am UKE Hamburg.

f.ufer@uke.de

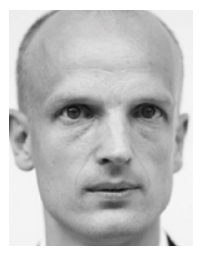

Dr. med. Mathias Gelderblom

ist Oberarzt an der Klinik und Poliklinik für Neurologie am UKE Hamburg. m.gelderblom@uke.de

Interessenkonflikt Die Autoren geben an, dass kein Interessenkonflikt besteht.

DOI 10.1055/s-0042-102289 Dtsch Med Wochenschr 2016; 141: 1239-1242 (c) Georg Thieme Verlag KG . Stuttgart · New York . ISSN 0012-0472
Genlokus | Bei der sehr viel selteneren hereditären neuralgischen Schulteramyotrophie konnte in einigen betroffenen Familien ein Lokus auf Chromosom 17q25 identifiziert werden. Jedoch unterstreicht die Tatsache, dass der Lokus in anderen Familien nicht nachweisbar war, die genetische Heterogenität dieser Entität.

Hepatitis E I Die Hepatitis E ist eine viral bedingte Leberentzündung, ausgelöst durch das HepatitisE-Virus (HEV) [3]. Während diese Infektion früher als Tropenkrankheit mit ausschließlich akutem Verlauf galt, hat sich diese Einschätzung in den letzten 10 Jahren geändert: HEV kommt nicht nur in den Tropen vor, sondern auch in Industrienationen und neben akuten Verläufen kann es bei Immunsupprimierten zu chronischen Verläufen kommen [3]. In den letzten Monaten wurde die Hepatitis E in Zusammenhang mit zahlreichen neurologischen Krankheitsbildern gebracht [4], u.a. der neuralgischen Schulteramyotrophie. Neben einzelnen Fallbeobachtungen [5-8], die eine neuralgische Schulteramyotrophie im Rahmen einer akuten Hepatitis E beobachteten, erregte Anfang 2014 eine in Neurology publizierte Studie großes Aufsehen: In einer Kohorte von 47 Patienten mit neuralgischer Schulteramyotrophie hatten $11 \%(n=5)$ simultan eine akute Hepatitis E [9].

Assoziation nicht ausreichend bekannt | Die NA ist eine mögliche extrahepatische Manifestation der Hepatitis E ( Tab. 1). Während die Hepatitis E als Differenzialdiagnose von Fällen unklarer Hepatitis den Gastroenterologen und Internisten geläufig ist, ist die Assoziation mit einer NA noch nicht ausreichend bekannt. Der Fall zeigt, dass autochthone, d.h. in Deutschland erworbene, HEV-Infektionen mögliche immunologische Trigger einer NA darstellen können.

Genese I Bislang ist die Genese der neuralgischen Schulteramyotrophie bei akuter Hepatitis E unklar. Aktuell werden neben einer direkten viralen Infektion von Strukturen des Plexus cervicobrachialis sekundäre immunologische Mechanismen diskutiert, die zu einer Schädigung des Plexus führen. Auch der präsentierte Fall kann den pathophysiologischen Zusammenhang nicht klären. Auffällig und klinisch wegweisend ist allerdings die bilaterale Beteiligung des Plexus cervicobrachialis. Während im Fall von Hepatitis E negativen NA nur $25-30 \%$ der Fälle eine bilaterale Beteiligung aufweisen, sind die Mehrzahl der Fälle mit Hepatitis E assoziierter NA bilateral [9].

Parallelen zum Guillain-Barré-Syndrom | Die bilaterale Beteiligung des Plexus wiederum stellt einen Zusammenhang zu einer weiteren neurologischen Komplikation einer Hepatitis-E-Infektion dar - dem Guillain-Barré-Syndrom (GBS). Vergleichbar mit einer bilateralen Plexusbeteiligung bei der Hepatitis-E-assoziierten NA verläuft auch das GBS bilateral. Außerdem sind bei beiden Er- krankungen primär Männer betroffen und die Häufigkeit einer akuten Hepatitis-E-Infektion ist ähnlich hoch ( $8-10 \%$ bei der NA und $5 \%$ beim GBS) $[9,10]$. Wie von van Eijk und Kollegen bereits diskutiert, kann es sich bei der Hepatitis-Eassoziierten NA und dem Hepatitis-E-assoziierten GBS somit um zwei klinisch verschiedene Syndrome handeln, die beide Ausdruck des Spektrums von viral getriggerten Entzündungsprozessen des peripheren Nervensystems sind $[9,10]$.

Kryoglobulinämie | Klassische immunologisch vermittelte Ursachen wie die Kryoglobulinämie konnten in diesem Fall zusätzlich ausgeschlossen werden, während zuvor ein Zusammenhang zwischen Kryoglobulinämie und HEV-Infektion aufgezeigt wurde $[11,12]$. Bald nach Verschwinden der HEV-Virämie sistierten bei dem hier präsentierten Patienten auch die Schmerzen und die Hypästhesie. Dies ist ein Indiz dafür, dass das Virus selber und nicht dadurch initiierte immunologische Prozesse pathophysiologisch ursächlich für die neuralgische Schulteramyotrophie sind.

Therapie mit Ribavirin | Jüngst zeigte eine retrospektive Fallserie an vier Patienten mit Hepatitis E und NA einen interessanten Aspekt hinsichtlich der möglichen Therapie der Hepatitis E mit Ribavirin auf. Während zwei dieser Patienten nicht behandelt worden waren, erhielten zwei Ribavirin und im weiteren Verlauf intravenöse Immunglobuline. Drei der dargestellten Patienten wiesen in Follow-Up Untersuchungen (16-126 Wochen später, Median 71 Wochen) weiterhin eine deutliche Schwäche auf. Während nur bei einem Patienten, der Ribavirin und intravenöse Immunglobuline erhielt, die Symptomatik komplett zurück gebildet war [13]. Somit ist der Stellenwert von Ribavirin weiterhin unklar. Da es sich bei dieser Therapie um Off-label-Medikation handelt, sollte dies nur in Einzelfällen und von Zentren mit ausreichender Erfahrung bzgl. Hepatitis-E-Therapie mit Ribavirin durchgeführt werden.

Konsequenz für Klinik und Praxis

- Die neuralgische Schulteramyotrophie (NA) kann in 8-10\% der Fälle mit einer akuten Hepatitis E assoziiert sein, so dass eine Testung auf HEV unbedingt ratsam ist.

- Der pathophysiologische Zusammenhang zwischen NA und Hepatitis E ist noch ungeklärt. Das Phänomen kann jedoch unabhängig von den klassischen Antikörpern auftreten.

- Um den Zusammenhang zwischen Hepatitis E und der NA besser zu verstehen, sammeln wir betroffene Patienten in einem Register und freuen uns, wenn man uns diese meldet.

\section{Literatur}

Vollständiges Literaturverzeichnis unter http://dx.doi.org/10.1055/s-0042-102289 
1 Pearce JM. Historical note. Neuralgic amyotrophy. Neurol Neurosurg Psychiatry 2005: 76: 389

2 van Alfen $\mathrm{N}$. The neuralgic amyotrophy consultation. J Neurol 2007: 254: 695-704

3 Pischke S, Behrendt P, Bock CT et al. Hepatitis E in Germany - an under-reported infectious disease. Dtsch Arztebl Int 2014: 111: 577-583

4 Cheung MC, Maguire J, Carey l et al. Review of the neurological manifestations of hepatitis $E$ infection. Ann Hepatol 2012: 11: 618-622

5 Decard BF, Grimm A, Andelova M et al. Hepatitis-E virus associated neuralgic amyotrophy with sustained plexus brachialis swelling visualized by high-resolution ultrasound. J Neurol Sci 2015: 351: 208-210

6 Moisset X, Vitello N, Bicilli E et al. Severe bilateral amyotrophic neuralgia associated with major dysphagia secondary to acute hepatitis E. F1000Res 2013: 2: 259

7 Theochari E, Vincent-Smith L, Ellis C. Neuralgic amyotrophy complicating acute hepatitis $\mathrm{E}$ infection: a rare association. BM] Case Rep 2015: 2015

8 Rianthavorn P, Thongmee C, Limpaphayom N, Komolmit P, Theamboonlers A, Poovorawan Y. The entire genome sequence of hepatitis $E$ virus genotype 3 isolated from a patient with neuralgic amyotrophy. Scand ] Infect Dis 2010: 42: 395-400

9 van Eijk JJ, Madden RG, van der Eijk AA et al. Neuralgic amyotrophy and hepatitis E virus infection. Neurology 2014: 82: 498-503

10 van den Berg B, van der Eijk AA, Pas SD et al. Guillain-Barre syndrome associated with preceding hepatitis E virus infection. Neurology 2014: 82: 491-497

11 Pischke S, Behrendt P, Manns MP, Wedemeyer $\mathrm{H}$. HEV-associated cryoglobulinaemia and extrahepatic manifestations of hepatitis $E$. Lancet Infect Dis 2014: 14: 678-679
12 Pischke S, Polywka S, Haag F et al. Association of hepatitis E virus and essential cryoglobulinemia? Journal of Clinical Virology 2015: epub ahead of print

13 Perrin HB, Cintas P, Abravanel F et al. Neurologic Disorders in Immunocompetent Patients with Autochthonous Acute Hepatitis E. Emerg Infect Dis 2015: 21

14 Kamar N, Bendall RP, Peron JM et al. Hepatitis E virus and neurologic disorders. Emerg Infect Dis 2011: 17: 173-179

15 Carli P, Landais C, Poisnel E et al. [Shoulder pain in a 30-year-old man]. Rev Med Interne 2012: 33: 111-114

16 Inghilleri ML, Grini Mazouzi M, Juntas Morales R. [Neuralgic amyotrophy as a manifestation of hepatitis E infection]. Rev Neurol (Paris) 2012: 168: 383-384

17 Peri AM, Milazzo L, Meroni L, Antinori S. Radiculoneuropathy associated with acute hepatitis $\mathrm{E}$. Dig Liver Dis 2013: 45: 963-964

18 Woolson KL, Forbes A, Vine L et al. Extra-hepatic manifestations of autochthonous hepatitis $\mathrm{E}$ infection. Aliment Pharmacol Ther 2014: 40: 1282-1291

19 Deroux A, Brion JP, Hyerle L et al. Association between hepatitis $\mathrm{E}$ and neurological disorders: two case studies and literature review. J Clin Virol 2014: 60: 60-62

20 Dartevel A, Colombe B, Bosseray A et al. Hepatitis E and neuralgic amyotrophy: Five cases and review of literature. J Clin Virol 2015: 69: 156-164

21 Martinez Rodriguez L, Carvajal P, Moris G. [Neuralgic amyotrophy associated to hepatitis $\mathrm{E}$ virus infection]. Med Clin (Barc) 2015: 145: 462-463

22 Fong F, Illahi M. Neuralgic amyotrophy associated with hepatitis E virus. Clin Neurol Neurosurg 2009; 111: 193-195 